\title{
The Medical Home Transformation
}

\author{
Namita Azad and Emily Hart \\ Montefiore Medical Group, 200 Corporate Blvd. South, Suite 175, Yonkers, NY 10701, USA
}

\begin{abstract}
To maintain productivity in today's healthcare market, it is important for institutions to have a delivery system that allows for transformation and adaptation. This paper introduces the Medical Home Transformation Model which demonstrates the effectiveness of employing a team of embedded coaches within a five-step capacity building program in conjunction with learning collaboratives, the adaptation medium. This model demonstrates the effectiveness of concurrently employing the coaching lifecycle and a five-step capacity building program. Moreover, the model details the formation of multidisciplinary teams at each clinical site, meaningful data reporting at site and physician levels and best practice sharing at collaboratives and seminars. A discussion then demonstrates the success of MMG (Montefiore Medical Group) as it applies the model to its ambulatory care network of 22 health centers in effort to achieve PCMH (Patient Centered Medical Home) recognition, improve clinical outcomes, and document financial benefits. While PCMH is currently the sought after delivery system framework, this paper will also demonstrate the adaptability of the model to any prescribed framework which continues advancement toward the "Triple Aim”. Such a model has enabled MMG to surpass national standards of clinical care within a confirmed vulnerable population and has placed MMG at the forefront for primary care delivery system reform.
\end{abstract}

Key words: Transformation, PCMH, primary care, quality improvement, innovation, coaching.

\section{Introduction}

The method by which healthcare is delivered to a consumer whether it be the individual, the family, or the community, is ever evolving. In attempt to remain current, healthcare systems receive constant scrutiny on the perceived impact of care from a myriad of reviewers. While the required changes in a delivery system are dynamic, there is consistent encouragement towards the evolution of the "Triple Aim”: improving the patient experience, improving the health of populations, and reducing the per capita cost of health care.

To maintain productivity in today's healthcare market, it is imperative for institutions to have a delivery system that allows for transformation and adaptation. Coaching, in its truest form, has provided a medium for topic experts to build capacity in novice apprentices catalyzing this transformation. The effectiveness of practice coaching has been well validated throughout the United States and other

Corresponding author: Namita Azad, MPH, research field: primary care transformation. countries with advanced health care systems. Kevin Grumbach et al. note in The Commonwealth Fund Issue Brief, "Facilitating Improvement in Primary Care: The Promise of Practice Coaching” states that coaching has demonstrated dramatic increases in preventative care measures (compared to control sites) by as much as $30 \%$. Grubach [1] eloquently summarizes that "successful strategies in the intervention included the coaching style of the practice improvement team, working alongside practice staff rather than just training them, and testing incremental changes using PDSA".

The missing link is the method for which a health care system can adapt and sustain the practice's transformation. This paper introduces the Medical Home Transformation Model which demonstrates the effectiveness of employing a team of embedded coaches within a five-step capacity building program in conjunction with learning collaboratives, the adaptation medium. A step-by-step guide demonstrates the applicability of the parallel processes in the model as well as best practice suggestions. A discussion then demonstrates the success of MMG 
(Montefiore Medical Group) as it applies the model to its ambulatory care network of 22 health centers in efforts to achieve PCMH (Patient Centered Medical Home) recognition. While PCMH is the current goal of the delivery system framework, this paper will also demonstrate the adaptability of the model to any prescribed framework which continues advancement toward the "Triple Aim”.

\section{Methodology}

The images displayed in Figs. 1 and 2 demonstrate the building blocks of creating capacity to implement a medical home. The embedded coaching team that employs the coaching lifecycle, is pivotal in sustainability of the medical home. The first step in this process, as represented by circle 1 in Fig, 1, speaks to increasing clinical and operational staffing inclusive of CCRN (Chronic Care Nurse Managers), PSR (Patient Service Representatives), and LPN (Licensed Practice Nurses) to manage sustainability of the transformation.

The second step is introducing an embedded coaching team that provides dedicated transformation support and who works with multidisciplinary care teams at the site level. The coaching team operates through a cyclical process displayed by the seven distinct steps in Fig. 2.

Steps of the coaching lifecycle:

(1) Needs Assessment: Conduct a site visit and meet with clinical and operational leadership to understand and identify gaps in patient flow. Review site specific data to gauge baseline site performance in areas of focus.

(2) Formation of Multidisciplinary Transformation Team: Form a multidisciplinary transformation team, representative of all site disciplines, which is responsible for planning, testing and leading site change and dissemination of information to all other site members.

(3) Vision and Strategy: Communicate rationale, define goals and align efforts.
(4) Workflow Design and Establishment of Metrics: Identify measure or area for improvement, outline and document the decided workflow.

(5) Create and Implement: Develop communication and roll-out plan for implementation of transformation activities across the site. Training occurs with all disciplines.

(6) Redesign \& Formalize: Guide transformation team to redesign and formalize workflows and their associated processes. This becomes part of the operational training for all new hires and annual competency review for existing associates.

(7) Sustain: Provide refresher training and revisit workflows on a continuous basis. Monitor site specific data and trends to assess fidelity to transformation activities.

Narrowing on key focus measures allows institutes to ensure progress towards system based health care goals. Circle 3 in Fig. 1, speaks to the necessity of data and analytics to support monitoring of identified focus measures for the transformation team at each site. Obtaining routine and real time data reports allows the team to work proactively on areas of opportunity, rather than reactively to historical data.

Care management and care coordination is the core of the PCMH delivery system. Circle 4 in Fig. 1, highlights the recruitment of co-located clinical staff such as health educators, certified diabetes educators, social workers and behavioral health staff or other clinical support staff relevant to the patient population. This comprehensive team ensures the patient and family is treated as a whole rather than narrowly focusing on one symptom or problem.

With the need to disseminate standardized workflows and processes, circle 5 in Fig. 1, identifies a platform such as a Transformation Collaborative which allows teams to work in collaboration with each other and create a shared language. This venue can be used for best practice sharing amongst the teams, content experts presenting and the introduction of new initiatives and programs. 


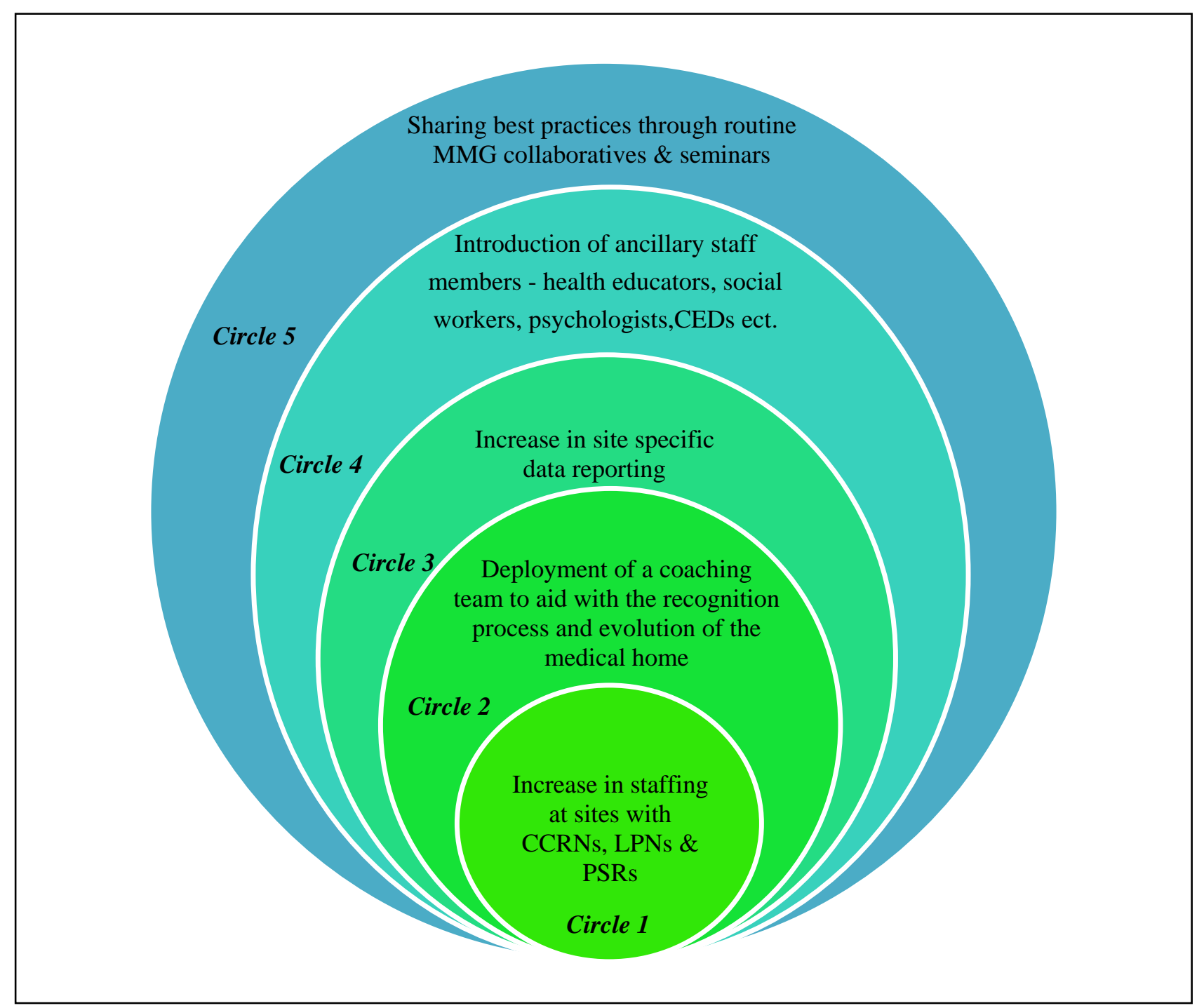

Fig. 1 The Medical Home Transformation Model.

Foundational to all this work is the science of CQI (Continuous Quality Improvement). MMG's vision is to create a CQI infrastructure to support success of the transformation work. Key CQI concepts such as PDSAs, Pareto Charts, Lean Wastes and Process Mapping, are taught at the Transformation Collaboratives and all sites are charged with creating a plan for a PDSA that they will test when they get back to the site. The coaching team then manages the follow-through with this as well as facilitates the dissemination of these concepts to the rest of the site staff. The utilization of these tools informs multiple steps of the coaching lifecycle such as steps 1 and 6, workflow design and redesign as well as step 5, create and implement.

\section{Results and Discussions}

The impact of this model is best demonstrated by the success within the MMG network. The population MMG serves is one of the most diverse in the country. In 2015, there were over 200,000 unique patients of which over $40 \%$ are Medicaid. MMG's transformation journey began in 2008 with two ambulatory practices that were selected as pilot PCMH sites and a team of two coaches were brought on board to initiate this transition. The coaches completed the needs 


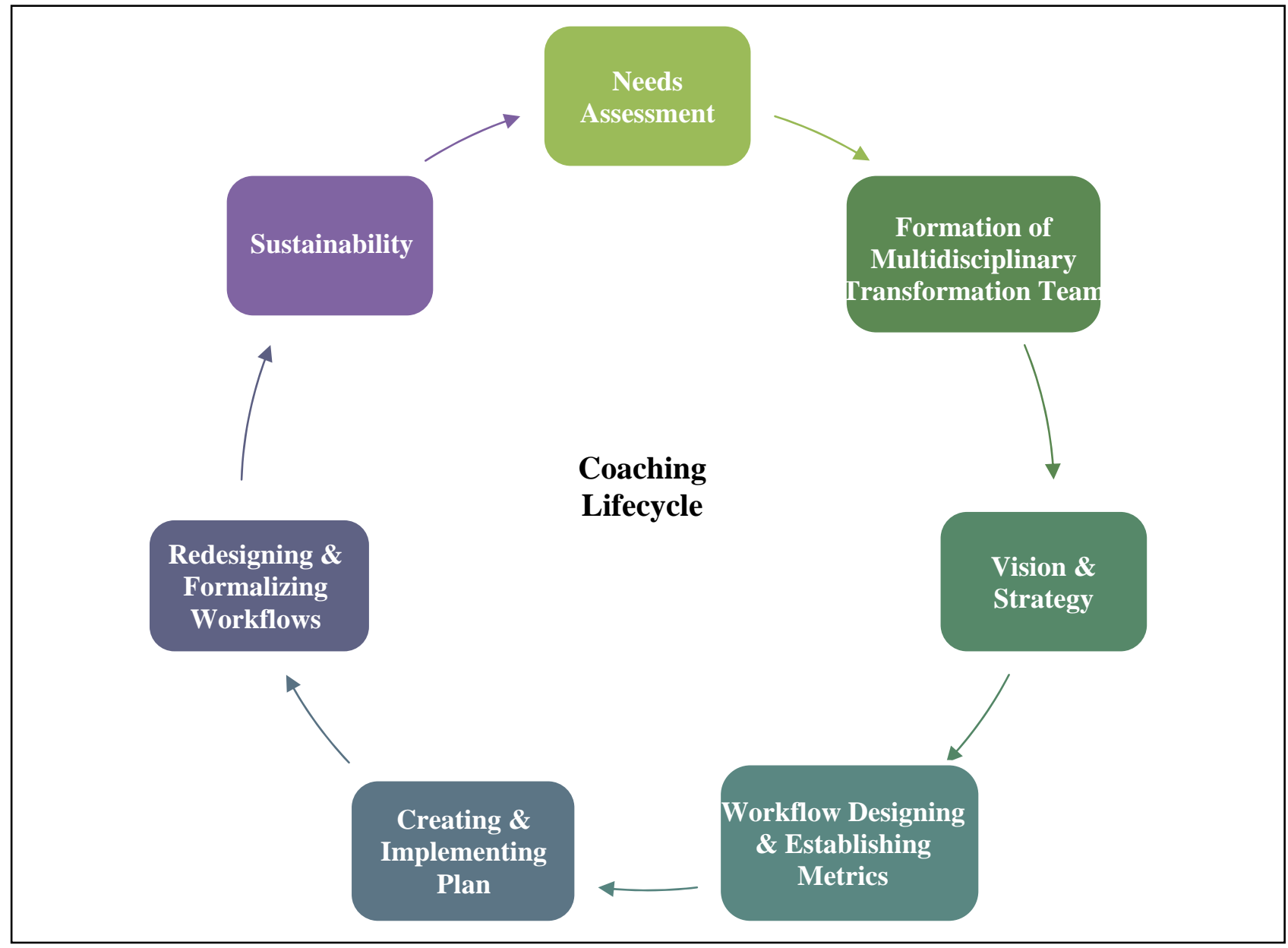

Fig. 2 Coaching lifecycle.

assessment at the two health centers and created the multidisciplinary teams. The needs assessment quickly identified the demand for additional support staff to create care management teams CCRNs, LPNs and PSRs were hired. These initial steps created the foundation for the transformation model, as displayed in Figs. 1 and 2. The outcome of utilizing this methodology was a level 3 PCMH certification under the 2008 standards for the two sites. The success seen from the model proved the need for replicating this staffing structure across the MMG network which included expansion of the coaching team.

Over the next 5 years, the MMG sites adopted the model and with additional coaching support, were able to achieve PMCH success under the 2008 and then 2011 standards. Through this period, the continual needs assessment led to formation of a robust reporting and analytics team, the necessity of having co-located service providers such as health educators, social workers, community health workers, psychologists, psychiatrists, patient educators and referral coordinators. The dynamic expansion of staffing and transformation activities required a forum for best practice sharing and learning that would facilitate dissemination on a larger scale. Quarterly held Transformation Learning Collaboratives were the product which brought interdisciplinary representatives together to learn tools and techniques as well as share their success and challenges. These pieces were the final building blocks to the current state of the transformation model.

An integral part of the medical home transformation model is the embedded coaching team that functions 


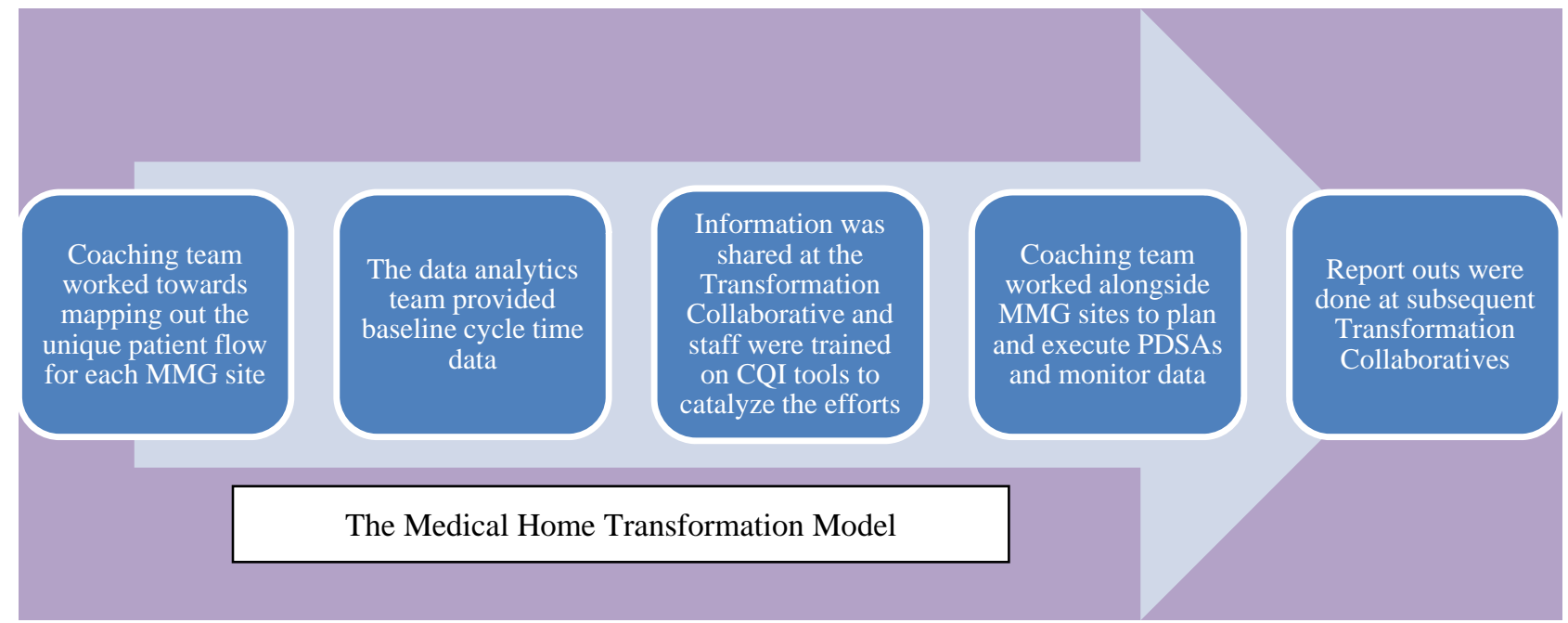

Fig. 3 MMG transformation example timeline.

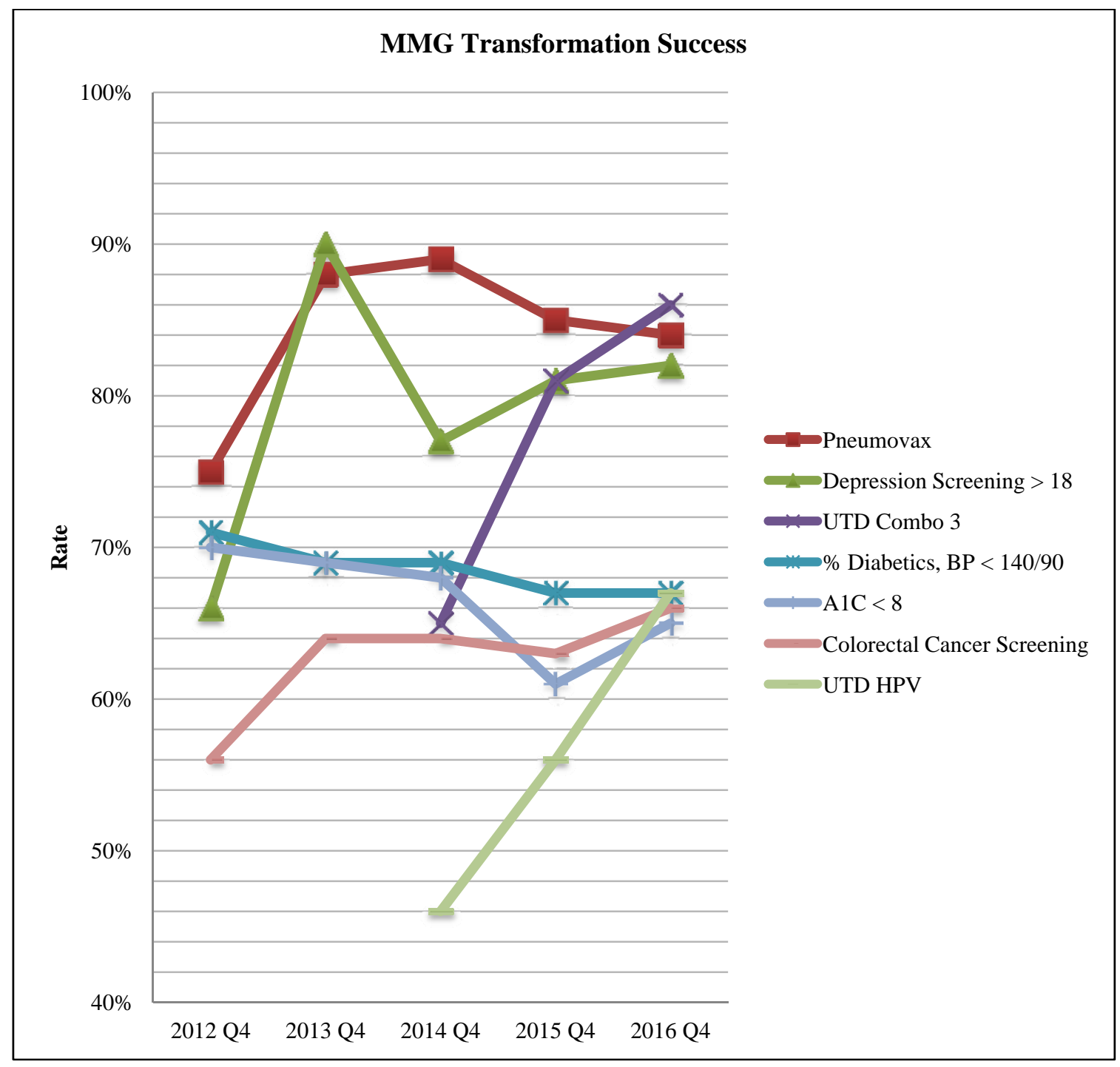

Fig. 4 MMG transformation success. 
as the catalyst in this process. As is highlighted in Fig. 2, the steps within the model allow for continuous monitoring and adaptation of site specific transformation activities based on clinical need.

The continuous utilization of the transformation model has fostered the sustainability of its PCMH standards since adoption. With the evolving standards of patience centered care, this model has enabled MMG to stay up to date with its certification as well as recertify the MMG sites under the 2014 standards. To date the 22 sites are certified under the 2011 standards of which 10 have been recertified as level 3 PMCHs under the 2014 standards.

With the MMG network functioning as a comprehensive $\mathrm{PCMH}$, there have been vast improvements in the clinical quality metrics. There have been numerous initiatives that have surfaced through different departments of MMG and the collaboration between the coaching team and these departments have led to success in the respective metrics of focus. One such collaboration was with MMG and the Montefiore CMO (Care Management Organization), the Hospital Medical Home grant, which led to a reduction in Medicaid readmission by 22\%. Other successes of the Medical Home Transformation Model over the last 4 years have included an increase in pneumovax vaccination rates by $9 \%$, increase in depression screening rates, for ages 18 and older by $16 \%$ and an increase in Combo 3 and HPV vaccination rates by $21 \%$.

The CQI knowledge building at the transformation collaboratives and its weaving into the coaching lifecycle has lent itself to the sustainability of this work. One of the metrics that this model has impacted is reduction of patient cycle time. Patient cycle time is a key component to the patience experience and access. MMG sites started this process by mapping out their current state patient flow map as a spaghetti diagram with baseline data (time in minutes) respective to each step. This helped them identify both value added and non-valued added steps and where the wastes lived. PDSAs were designed to start working on each of the wastes identified. Numerous PDSA cycles were conducted and in a 6-month period, more than $75 \%$ of MMG health centers were able to reduce their patient cycle time by $10 \%$ which accounted to over $40 \mathrm{~min}$ in some health centers. There was a steady improvement in the patient experience scores in the domains of physician communication and care coordination. One of the critical factors to team based care, as per the PMCH 2014 standards, is the utilization of huddles. This practice proved to be one of the key drivers in reduction of patient cycle time. Site staff used the huddles as the venue to do pre-visit planning for patients. Some health centers conducted huddles twice a day-the first being done in the morning to plan the operational needs of that day and the second being done at the end of the day to plan the clinical needs of the following day. This process helped facilitate targeted and coordinated care for the patient.

Fig. 2 below demonstrates how the Medical Home Transformation Model was the framework that allowed for this success.

\section{Conclusions}

The Medical Home Transformation Model has provided MMG with the capacity to create macro level cultural changes, exhibited through its team based care delivery which operates within a solid CQI infrastructure. This has enabled MMG to surpass national standards of delivered clinical care within a highly vulnerable population and has placed this health system at the forefront for primary care delivery system reform.

The anticipated reform of primary care will bring responsibility to institutes to address more than just the patient's current medical condition but rather look at the patient and their community as a whole. Moving forward, MMG's vision is to maximize the level of 
patient engagement and satisfaction through optimal tools such as electronic patient portals, shared patient health information platforms and patient and community advisory councils.

\section{Reference}

[1] Grumbach, K., Bainbridge, E., and Bodenheimer, T. 2012. Facilitating Improvement in Primary Care: The Promise of Practice Coaching. The Commonwealth Fund, 1-13. 